\title{
Mathematical model to determine the effect of a sub-glycocalyx space
}

\author{
Mohit P. Dalwadi $\odot,{ }^{1,2, *}$ John R. King $\odot{ }^{2,3}$ Rosemary J. Dyson $\odot,{ }^{4}$ and Kenton P. Arkill ${ }^{5, \dagger}$ \\ ${ }^{1}$ Mathematical Institute, University of Oxford, Oxford, OX2 6GG, United Kingdom \\ ${ }^{2}$ Synthetic Biology Research Centre, University of Nottingham, Nottingham, NG7 2RD, United Kingdom \\ ${ }^{3}$ School of Mathematical Sciences, University of Nottingham, Nottingham, NG7 2RD, United Kingdom \\ ${ }^{4}$ School of Mathematics, University of Birmingham, Birmingham, B15 2TT, United Kingdom \\ ${ }^{5}$ School of Medicine, University of Nottingham, Nottingham, NG7 2UH, United Kingdom
}

(Received 26 April 2019; accepted 17 January 2020; published 27 April 2020)

\begin{abstract}
We consider the drainage of blood plasma across the capillary wall, focusing on the flow through the endothelial glycocalyx layer that coats the luminal surface of vascular endothelial cells. We investigate how the presence of a sub-glycocalyx space between the porous glycocalyx and the impermeable endothelial cells affects the flow, using the Darcy and Stokes equations to model the flow in the glycocalyx and sub-glycocalyx space, respectively. Using an asymptotic analysis, we exploit the disparity of length scales to reduce the problem complexity to reveal the existence of several asymptotic regions in space. We provide a detailed characterization of the flow through the glycocalyx layer in terms of the microscale system parameters, and we derive analytic macroscale results, such as for the flux through and hydraulic conductivity of the glycocalyx layer. We show that the presence of a sub-glycocalyx space results in a higher flux of blood plasma through the glycocalyx layer, and we use our theoretical predictions to suggest experiments that could be carried out to shed light on the extent of the layer.
\end{abstract}

DOI: 10.1103/PhysRevFluids.5.043103

\section{INTRODUCTION}

The endothelial glycocalyx (eGlx) is a porous coating found on the luminal surface of most blood vessels $[1,2]$. The eGlx is a complex biochemical and structural entity, and though there is little consensus within the field over the specifics of its biochemical make-up and structure, it is known to serve several purposes: it acts as a molecular sieve for plasma proteins; it regulates blood cell interactions; it acts as a mechanotransducer of hydrodynamical shear stress to the endothelial cells to which it is attached; and it modulates the vascular permeability of the vessel wall to plasma leakage to the interstitium [1,3-6]. Damage to the vasculature through inflammation, disease, or insult can change the local resistance of the eGlx structure and hence affect the drainage of plasma [7-9]. To aid the use of targeted therapies, it is important to develop a better understanding of how the microstructure of the eGlx layer affects the function of the vascular wall.

As blood plasma drains from the lumen on its way to the interstitium, it first passes through the porous eGlx attached to the endothelial cells. The impermeability of the endothelial cell body

\footnotetext{
*mohit.dalwadi@maths.ox.ac.uk
}

${ }^{\dagger}$ kenton.arkill@ nottingham.ac.uk

Published by the American Physical Society under the terms of the Creative Commons Attribution 4.0 International license. Further distribution of this work must maintain attribution to the author(s) and the published article's title, journal citation, and DOI. 


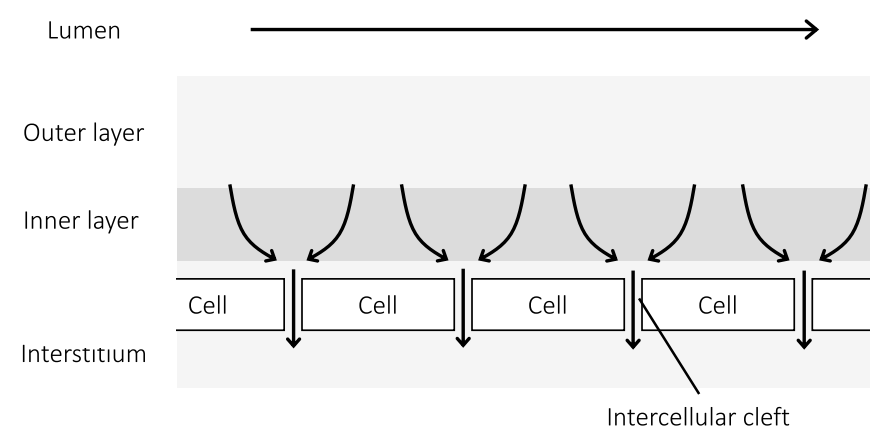

FIG. 1. A schematic of the endothelial glycocalyx and the plasma leakage through it as a cross section through the radial and axial directions of the capillary. As blood passes through the lumen, a small amount of plasma leaks across the capillary wall. This plasma travels through the two-layer structure of the eGlx and then down the intercellular clefts between neighboring endothelial cells before reaching the interstitium.

means that in nonfenestrated vessels the only route to the interstitium is though the small gaps between these cells, referred to as the intercellular clefts [4]. The current belief is that the the eGlx is a layered structure consisting of an inner layer (approximately $200 \mathrm{~nm}$ thick) which acts as a filtration barrier and a highly permeable diffuse outer layer (up to $1000 \mathrm{~nm}$ thick) which is involved in the mechanotransduction and mechanistic roles [10]. We provide a schematic of the structure of, and the flow through, the eGlx in Fig. 1.

The global resistance of the vascular wall can be experimentally measured using single capillary cannulation with pressure control [11,12], but determining the microstructure of individual layers is more difficult. Electron microscopy is currently the only experimental method with the potential to determine the ultrastructural space in the glycocalyx structure. Although there is some visual evidence for a sub-glycocalyx space $[13,14]$, the extent of this space is unclear due to the practicalities of the imaging process, and several challenges with this procedure remain. First, when using electron microscopy, the eGlx must be stained and immobilized since polysaccharides (which make up part of the glycocalyx) are not fully immobilized by aldehyde fixation [15-18]. These charged stains induce a structural alteration of the glycocalyx, including a collapse of the layer. Second, when curing the resin between the tissue and the lumen, splitting between the fixed cells in the tissue and the relatively empty lumen can occur, making it difficult to validate the extent of this sub-glycocalyx space experimentally.

It is particularly important to understand the extent of this space for the revised Starling hypothesis. The standard Starling hypothesis provides a macroscale relationship between the plasma flux and the net driving pressure between the lumen and the interstitium [19]. However, common misinterpretations of the hypothesis do not agree with physiological experiments due to confusion over the nature of the oncotic pressure from albumin concentration [20], which usually acts in the opposite direction to hydrostatic pressure [21,22]; while varying the albumin concentration in the lumen alters the flux as expected, varying the albumin concentration in the interstitium does not [22].

This discrepancy is explained by the revised Starling hypothesis, which proposes that there is a critical point below the eGlx but above the interstitium wherein the albumin concentration is negligible. Moreover, it holds that the important locations for calculating the oncotic pressure difference are between the lumen and this critical point, rather than the lumen and the interstitium. The oncotic pressure difference between the critical point and the interstitium can then be ignored, as the flow through the intercellular clefts to the interstitium is rapid enough to negate diffusion back from the tissue [20,23,24]. However, the location of this critical point is not known precisely, largely due to the unknown nature of the eGlx structure. As the filter functionality of the eGlx means that the critical point is likely to be immediately below the eGlx, it is vital to know whether 
(a)

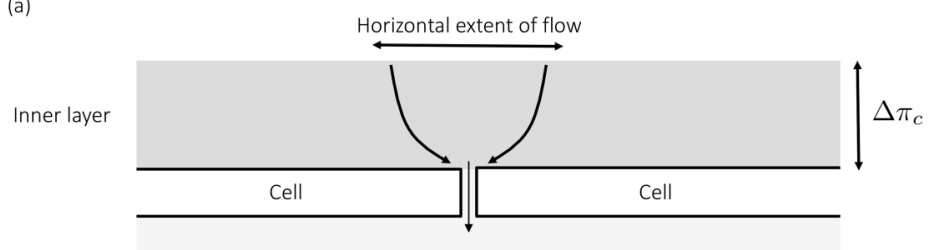

(b)

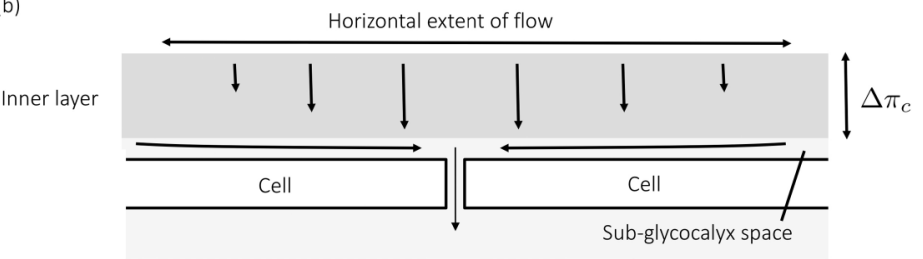

FIG. 2. A schematic of the differences between the plasma leakage through the glycocalyx, centered around a single intercellular cleft when there is (a) a negligible sub-glycocalyx space (Case 1) and (b) a significant subglycocalyx space (Case 2). The question of the existence of a sub-glycocalyx gap has important implications for calculating the oncotic pressure $\left(\Delta \pi_{c}\right)$ across the glycocalyx in the revised Starling hypothesis. In this paper, we show that the horizontal extent of the flow (and thus the useful filtration width) in the glycocalyx is larger when the sub-glycocalyx space is significant, i.e., in Case 2.

the eGlx terminates above the endothelial cells to leave a significant sub-glycocalyx space or much closer to the level of the endothelial cells where the intercellular clefts begin. This is because the former possibility has a much greater seepage area than the latter, with important implications for the revised Starling hypothesis, since this gives the plasma flux per unit area.

The main goal of this paper is to determine the effect of any sub-glycocalyx space on the flow through the eGlx and whether the height of this space can be inferred through future experiments. This space is not necessarily devoid of all material, as the protein core tether would attach the porous glycocalyx to the surface of the endothelial cells, but it would have a very large void fraction, in contrast to the filtration zone of the eGlx. The size and extent of this sub-glycocalyx space are unknown: it could range from being a small region localized around the entrance of each cleft through to extending under the entire glycocalyx. We compare these two extreme scenarios in this paper and provide a schematic of them in Fig. 2. In addition, understanding the flow though the eGlx will allow us to determine the horizontal extent of the flow within the glycocalyx, as well as to derive the macroscale hydraulic conductivity, a constitutive parameter in the revised Starling hypothesis, as a function of the microscale properties of the eGlx.

Mathematical modeling has been used to understand the role of the glycocalyx in molecular transport better, with a focus on the effect of the junction strands within the intercellular clefts. The most extensive models are combined flow and transport models due to Weinbaum and colleagues [25-27]. While these models are useful, they do not address the issue of a sub-glycocalyx space or the horizontal extent of the flow within the glycocalyx (as indicated in Fig. 2) and hence the proportion of the glycocalyx that is used for filtration. In the recent Ref. [28], the authors consider the coupled solute transport and flow problem across microvessel walls, with the aim of investigating solute exchange with time-dependent pressure drops across the wall. However, as the authors model the endothelial glycocalyx as a membrane, the exact nature of the flow within the endothelial glycocalyx is not considered.

In this paper, we seek to investigate the effect of a sub-glycocalyx space on the flow through the glycocalyx. To focus on the geometrical effect of the sub-glycocalyx space, we do not consider solute transport. We consider the flow through the glycocalyx in two cases: where the height of the 
sub-glycocalyx space is comparable to or much larger than the typical pore size within the porous glycocalyx. We refer to these two cases as the height of the sub-glycocalyx space being "negligible" and "significant", respectively. We assume that the glycocalyx and sub-glycocalyx space each has a uniform height and spans the entirety of the vessel wall, including over the intercellular clefts. To focus on the effect of a sub-glycocalyx space on the flow, we choose a simplified model geometry in which the cross section of an endothelial cell is rectangular, resulting in right-angled corners at the entrance to the intercellular cleft and no junction strand within the cleft. In addition, we will also neglect the effect of the highly porous diffuse eGlx region, which we do not expect to have a significant effect on the plasma leakage [10], and of any tight junctions or fenestrations in our vasculature. The latter assumptions mean that our model will be suitable only for non-neural and nonglomerular vasculature. There has been modeling work for the case with a junction strand but without a sub-glycocalyx [29] and, more recently, for the flow through the bilayer structure [10].

As the glycocalyx has a complex bushy structure, we model the plasma flow through this region using the Darcy equations for flow through a porous medium. The intercellular cleft and the (speculative) sub-glycocalyx space do not contain these resistive structures, and so we model the flow in these regions using Stokes equations. There are several important questions to consider when coupling these two flow regions. One such is the correct interfacial conditions to impose on a boundary between Stokes and Darcy flow regions, which remains an area of active research [30,31]. We use the boundary conditions of continuity of pressure, continuity of flux, and a Beavers-Joseph slip condition. The first two conditions were derived in Ref. [32] and the last was experimentally derived in Ref. [33], and given theoretical justification in Ref. [34]. Another important question is whether the two regions should be treated as distinct. If the height of the sub-glycocalyx space is of the same order as the pore size within the glycocalyx, the sub-glycocalyx space is indistinguishable from the porous glycocalyx region and thus should be governed by Darcy's equations. As we will show, neither the case with a sub-glycocalyx space nor the case without is a sublimit of the other, and thus we must consider two potential models: the first where there is a negligible sub-glycocalyx space (any such space is then indistinguishable from the Darcy region), and the second where there is a significant sub-glycocalyx space, resulting in a separate sub-glycocalyx region.

The structure of this paper is as follows. In Sec. II we present the general dimensional problem, which accounts for both cases (i.e., when the sub-glycocalyx space is significant and negligible). In Sec. III we specifically consider the case with a negligible sub-glycocalyx space; we nondimensionalize the problem and then perform an asymptotic analysis by exploiting the small aspect ratio of the geometry, allowing us to derive analytic solutions for the flow in each asymptotic region. In Sec. IV we consider the case with a significant sub-glycocalyx space, and the analysis proceeds in a similar manner to that described above. In Sec. V we summarize the main differences between the cases, and, finally, in Sec. VI we discuss our results and their physical implications.

\section{MODEL SETUP}

We consider the leakage of plasma across the endothelial glycocalyx layer, from the boundary between lumen and glycocalyx to the interstitial fluid located beyond the endothelial cells. We treat the bushy glycocalyx as a porous medium and the endothelial cells to which it attaches as impermeable boundaries. The plasma leaks through the gaps between endothelial cells, referred to as intercellular clefts, into the reservoir of interstitial fluid located below the endothelial cells.

As an intercellular cleft has typical width much smaller than the typical radius of an endothelial cell and is not particularly tortuous, we simplify the geometry by considering the two-dimensional problem with the intercellular cleft perpendicular to the lumen wall. Essentially, we neglect any effect of cell curvature or of junctions where intercellular clefts meet. In addition, we consider the problem with one intercellular cleft in an infinite domain. Although there are many intercellular clefts along the glycocalyx in the lumen (see Fig. 1), we will show that the flow decays exponentially away from the intercellular clefts sufficiently rapidly that restricting our model to a single intercellular cleft has no significant bearing on the results. Additionally, we will not consider the 


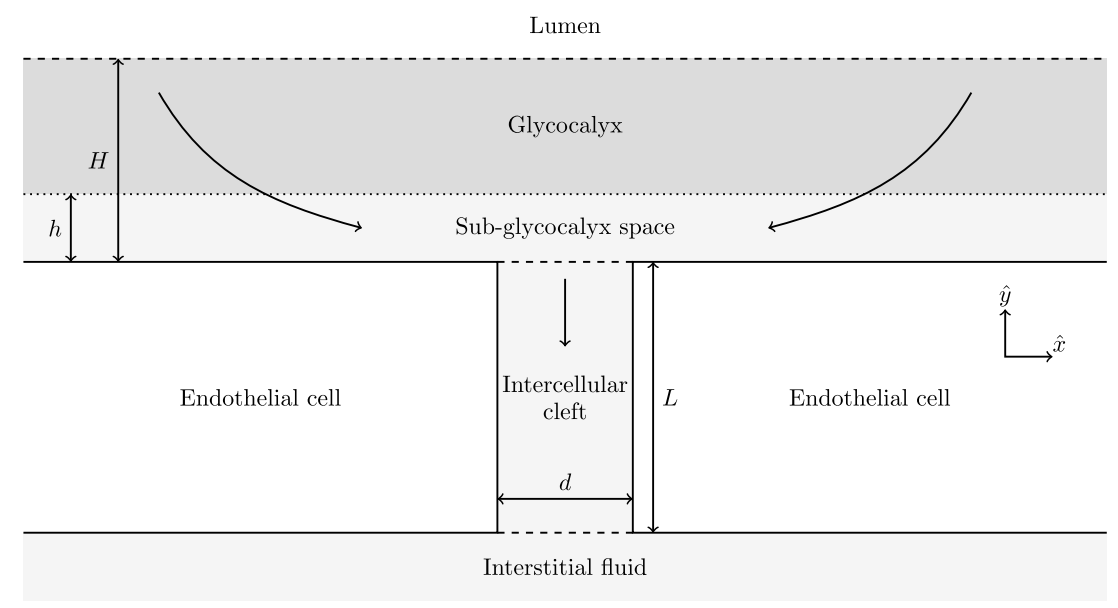

FIG. 3. A schematic of the model geometry. The single-headed arrows show the flow direction. It is not known whether the sub-glycocalyx exists, and so we consider $h \geqslant 0$. As explained in this paper, we must consider different models for $h=0$ and $h>0$.

flow within the main blood vessel above the glycocalyx, instead imposing a constant pressure difference across the system, which extends from the interface between the glycocalyx and the lumen at the top to the end of the intercellular cleft at the bottom. This will allow us to obtain a relationship between the total pressure drop across and the flux through the system. Finally, we also assume that the lumen to glycocalyx, and glycocalyx to sub-glycocalyx-space interfaces are a uniform distance from the endothelial cell membrane.

We work in $(\hat{x}, \hat{y})$ coordinates, which are tangential and perpendicular to the lumen wall, respectively, and we show a schematic of the model setup in Fig. 3. We model plasma flow within the glycocalyx as incompressible Darcy flow

$$
\hat{\boldsymbol{U}}=-\frac{k}{\mu} \nabla \hat{P}, \quad \nabla \cdot \hat{\boldsymbol{U}}=0 \quad \text { for } \hat{x} \in \mathbb{R}, \hat{y} \in(h, H),
$$

with velocity $\hat{\boldsymbol{U}}=(\hat{U}, \hat{V})$ and pressure $\hat{P}$, through a porous medium with constant permeability $k$. As the plasma moving through the glycocalyx does not contain blood cells, we treat it as an incompressible Newtonian fluid with constant viscosity $\mu$. We model the flow in the sub-glycocalyx space and in the intercellular cleft as incompressible Stokes flow

$$
\nabla \hat{p}=\mu \nabla^{2} \hat{\boldsymbol{u}}, \quad \nabla \cdot \hat{\boldsymbol{u}}=0 \quad \text { for } \hat{x} \in \mathbb{R}, \hat{y}=(0, h) \text { and }|\hat{x}|<d / 2, \hat{y}=(-L, 0),
$$

with velocity $\hat{\boldsymbol{u}}=(\hat{u}, \hat{v})$ and pressure $\hat{p}$. The entire glycocalyx and sub-glycocalyx structure has height $H$, the sub-glycocalyx has height $h$ (so $0 \leqslant h<H$ ), and the intercellular cleft has width $d$. As discussed in Sec. I, the height and extent of the sub-glycocalyx space are largely unknown. The space could range from only existing at the cleft entrance (the case $h=0$ ) to extending across the entire endothelial cell membrane (the case $h>0$ ). In the latter case, the glycocalyx is held away from the endothelial cells by thin stems. As a modeling assumption, we neglect any effect of these stems on the flow, and we will show that the aspect ratio of this problem results in lubrication scalings within the sub-glycocalyx space. Finally, as mentioned in Sec. I, when the sub-glycocalyx height or the intercellular cleft width is comparable to the typical pore size in the glycocalyx, the two regions are indistinguishable. Mathematically, these occur when $h=k^{1 / 2}$ and $d=k^{1 / 2}$, respectively. In this case, the Darcy flow equations govern all the flow above the intercellular cleft. We will show that the limit $h \rightarrow 0$ is singular and does not yield the same results as for the case where $h=0$. 
At the interface between the Darcy and the Stokes flow regions, we impose continuity of pressure, continuity of flux, and a Beavers-Joseph slip condition for the tangential velocity in the sub-glycocalyx region [32,33] as follows:

$$
\hat{P}=\hat{p}, \quad \hat{V}=\hat{v}, \quad \frac{a}{\sqrt{k}}(\hat{U}-\hat{u})=\frac{\partial \hat{u}}{\partial \hat{y}} \quad \text { on } \Gamma,
$$

where $a$ is the dimensionless Beavers-Joseph slip coefficient and $\Gamma$ is the interface between the Darcy and Stokes regions. We emphasize that the Beavers-Joseph slip condition will not be appropriate if there is an impermeable membrane in the Stokes region close to the permeable interface (as would occur in the limit $h \rightarrow 0$ ), and, as such, we define this interface separately for the two cases we consider. At the boundary where the intercellular cleft region meets the endothelial cells, we impose a no-slip condition

$$
\hat{\boldsymbol{u}}=\mathbf{0} \quad \text { for }|\hat{x}|=d / 2, \quad \hat{y} \in(-L, 0) .
$$

We note that when there is no sub-glycocalyx space $(h=0)$, we will require an additional no-flux condition at the interface between the glycocalyx and the endothelial cells, whereas when there is a sub-glycocalyx space $(h>0)$, we will require an additional no-slip condition at the interface between the sub-glycocalyx-space and the endothelial cells. We will introduce both of these later when required. Finally, we impose a constant pressure difference of $\Pi$ across the system as follows:

$$
\begin{aligned}
& \hat{P}=\Pi \quad \text { for } \hat{x} \in \mathbb{R}, \quad \hat{y}=H, \\
& \hat{p}=0 \quad \text { for }|\hat{x}|<d / 2, \quad \hat{y}=-L .
\end{aligned}
$$

It will also be helpful to use our model to calculate the steady fluid flux through the glycocalyx system, defined as

$$
Q=-\int_{-d / 2}^{d / 2} v(\hat{x},-L) d \hat{x}
$$

as a function of the pressure drop. To quantify this relationship, we define the effective resistance

$$
K=\frac{\Pi}{\mu Q} \text {. }
$$

We give typical parameter values for this system in Table I and note that there are several extreme parameter ratios; exploiting these will facilitate our analysis. In particular, we note that $H \ll D$, the height of the glycocalyx is much smaller than the typical length scale of an endothelial cell; $d \ll L$, the width of the intercellular cleft (the endothelial gap) is much smaller than the length of the cleft; and $d \ll H$, the width of the cleft is much smaller than the height of the glycocalyx. Finally, we also note that $k \ll d^{2}$, corresponding to the typical pore size in the glycocalyx being smaller than the width of the intercellular cleft, and thus, as discussed in Sec. I, the Stokes equations are valid within the intercellular cleft. In the two cases we consider, with and without a sub-glycocalyx space, we will first nondimensionalize the problem appropriately, and then exploit these extreme ratios to solve the system. We start by considering the problem with no sub-glycocalyx space.

\section{CASE 1: NEGLIGIBLE SUB-GLYCOCALYX SPACE}

We first consider the problem with no sub-glycocalyx space, corresponding to $h=0$, where the glycocalyx extends directly to the endothelial cells. With the addition of the no-flux condition

$$
\hat{V}=0 \quad \text { for }|\hat{x}|>d / 2, \quad \hat{y}=0,
$$

at the boundary where the glycocalyx meets the endothelial cells, the governing system for Case 1 is defined by (1)-(5) and (8). Additionally, we note that $\Gamma$, the interface for (3), is defined as

$$
\Gamma=\{(\hat{x}, \hat{y}):|\hat{x}|<d / 2, \hat{y}=0\},
$$


TABLE I. Approximate values of the system parameters. The height of the glycocalyx could vary from anywhere between 150 and $400 \mathrm{~nm}$ [4]. The approximate porosity of the glycocalyx is estimated by treating the glycocalyx as a hexagonal lattice of fibers with center-to-center fiber separation of $20 \mathrm{~nm}$ and fiber radius of $6 \mathrm{~nm}$ [27]. The height of the sub-glycocalyx space is unknown, though it has been observed and is expected to be between $0-50 \mathrm{~nm}$ [14]. For the permeability, we use the approximation $k \approx \phi d_{p}^{2} / 96$ from Sec. 5.10 of Ref. [36] for a porous medium consisting of randomly orientated equal circular tubes of diameter $d_{p}$.

\begin{tabular}{lcc}
\hline \hline Variable & Description & Approximate value \\
\hline$H$ & Height of glycocalyx layer & $200 \mathrm{~nm}[4]$ \\
$d_{p}$ & Pore width within glycocalyx & $8 \mathrm{~nm} \mathrm{[22]}$ \\
$\phi$ & Porosity of glycocalyx & $0.7[27]$ \\
$h$ & Height of sub-glycocalyx space & $40 \mathrm{~nm}$ (assumed) \\
$D$ & Length of endothelial cell & $4000 \mathrm{~nm}[25]$ \\
$L$ & Height of intercellular cleft & $400 \mathrm{~nm}[25]$ \\
$d$ & Width of intercellular cleft & $10 \mathrm{~nm} \mathrm{[25]}$ \\
$\Pi$ & Hydraulic pressure difference across system & $3 \mathrm{~cm} \mathrm{H}_{2} \mathrm{O}[27]$ \\
$\mu$ & Dynamic viscosity of plasma & $1.2 \mathrm{mPa} \mathrm{s}^{2}[35]$ \\
$k$ & Permeability of glycocalyx & $0.5 \mathrm{~nm}^{2}[36]$ \\
$a$ & Beavers-Joseph slip coefficient & $\mathrm{Unnnown}^{-}$ \\
\hline \hline
\end{tabular}

for Case 1. We give a schematic of our model setup and summarize the governing system in Fig. 4.

\section{A. Dimensionless problem}

We scale our variables as follows:

$$
(\hat{U}, \hat{V}, \hat{u}, \hat{v})=\frac{k \Pi}{\mu H}(U, V, u, v / \delta), \quad(\hat{P}, \hat{p})=\Pi(P, p), \quad(\hat{x}, \hat{y})=H(x, y),
$$

where $\delta=d / 2 H$. From (1), we obtain the dimensionless problem in the glycocalyx

$$
U=-P_{x}, \quad V=-P_{y}, \quad U_{x}+V_{y}=0 \quad \text { for } x \in \mathbb{R}, y \in(0,1),
$$

and from (2), in the intercellular cleft

$$
p_{x}=\delta^{2} \omega \nabla^{2} u, \quad p_{y}=\delta \omega \nabla^{2} v, \quad \delta u_{x}+v_{y}=0 \quad \text { for }|x|<\delta, y \in(-\lambda, 0),
$$

where $\omega=4 k / d^{2}$ and $\lambda=L / H$. Finally, the boundary and coupling conditions (3)-(5) and (8) become

$$
\begin{aligned}
& P=1 \quad \text { for } x \in \mathbb{R}, y=1, \\
& v=\delta V, \quad p=P, \quad U-u=\delta \alpha \frac{\partial u}{\partial y} \quad \text { for }|x|<\delta, y=0, \\
& V=0 \quad \text { for }|x|>\delta, y=0, \\
& \boldsymbol{u}=\mathbf{0} \quad \text { for }|x|=\delta, y \in(-\lambda, 0), \\
& p=0 \quad \text { for }|x|<\delta, y=-\lambda,
\end{aligned}
$$

where $\alpha=2 k^{1 / 2} / a d$.

\section{B. Asymptotic structure}

To facilitate our analysis, we exploit the small parameter ratios in the system and consider the asymptotic limits of $k \ll d^{2}$ and $d \ll H$, corresponding to $\omega \rightarrow 0^{+}$and $\delta \rightarrow 0^{+}$. We proceed by performing an asymptotic analysis in these two small parameters, and we will find that there is 


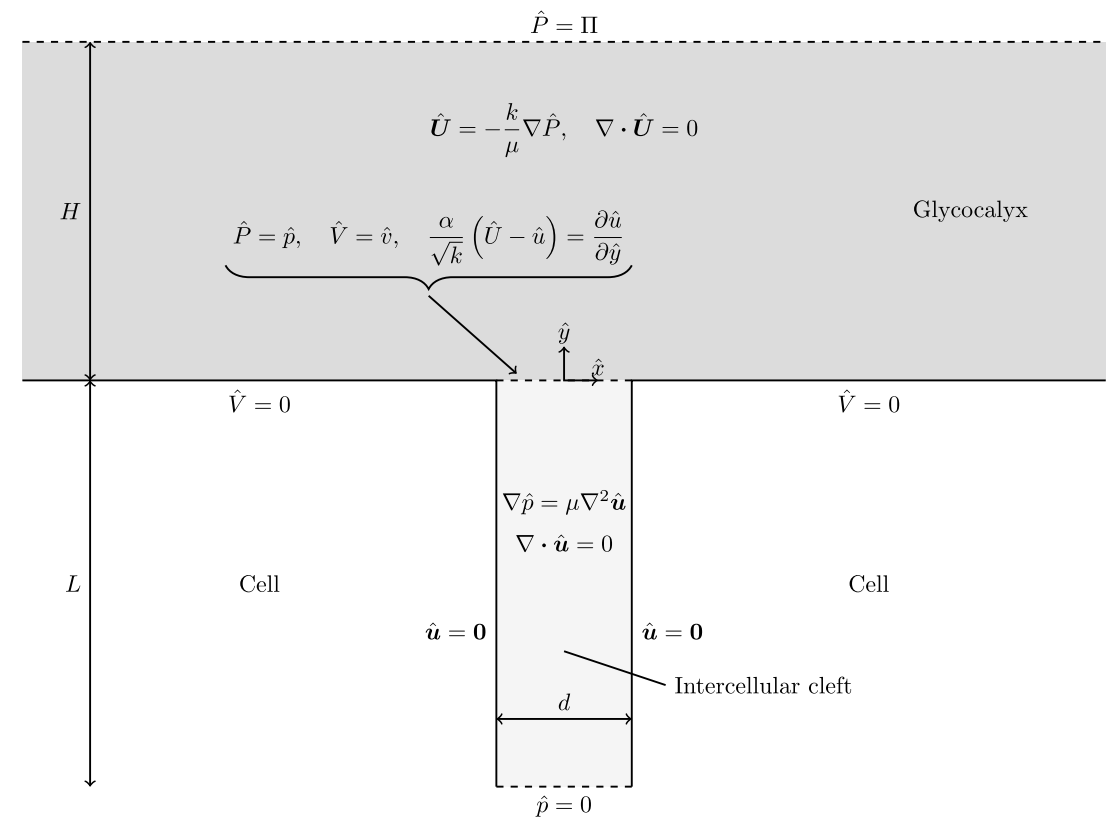

FIG. 4. Schematic of the dimensional flow problem with no sub-glycocalyx space. Darcy flow governs the dark gray region, Stokes flow governs the light gray region, and the white regions denote the impermeable endothelial cells.

a distinguished asymptotic limit when $\omega=O(\delta)$. Hence, we introduce $\omega=\delta \xi$, where $\xi=O(1)$. Additionally, we take $\lambda$ to be of $O(1)$, essentially a statement that the height of the glycocalyx is of the same order as the length of the intercellular cleft, and from this we also implicitly consider the limit $d \ll L$. The magnitude of $a$, the Beavers-Joseph slip coefficient, is relatively unknown, so we take $\alpha=2 k^{1 / 2} / a d$ to be of $O(1)$ in Case 1 , which results in a distinguished asymptotic limit from the tangential slip condition. We will find, however, that this dimensionless grouping has no effect on the leading-order flux through the glycocalyx for this case.

We illustrate the asymptotic structure of the problem in Fig. 5. The intercellular cleft (Region III) acts as a sink to the flow in the glycocalyx. In Region I, the channel acts as a point sink, and thus the flow magnitude is of the same asymptotic order in both horizontal and vertical directions. In Region II the finite nature of the intercellular cleft becomes apparent, and, although the flow velocity is increased by a factor of $1 / \delta$ in Region II over that in Region I, the flow magnitude is of the same asymptotic order in both horizontal and vertical directions. For notational purposes, we split Region II into the glycocalyx (Region IIa) and the intercellular cleft (Region IIb). As the flow from Region II carries on vertically downwards into Region III, the flow magnitude in Region III is the same as in Region II, though it is directed vertically at leading order.

\section{Solving for the flow}

\section{Region I}

We start by considering the asymptotic limit $\delta \rightarrow 0^{+}$. As the intercellular cleft acts as a sink, the effective leading-order problem in Region I is

$$
\nabla^{2} P=2 \kappa \hat{\delta}(\boldsymbol{x}),
$$

where $\kappa$ is a measure of the dimensionless sink strength, which will be obtained later via matching, and $\hat{\delta}(\boldsymbol{x})$ is the Dirac delta function. We use the hat to distinguish the Dirac delta function $\hat{\delta}$ from 


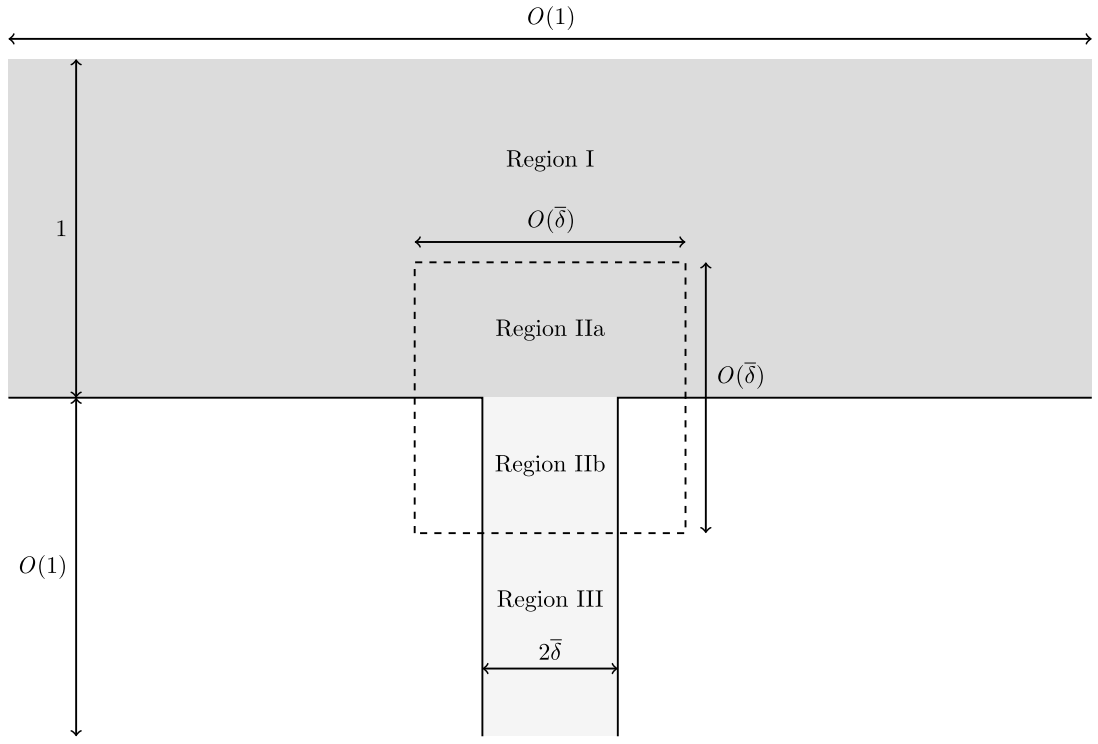

FIG. 5. The asymptotic structure of the problem in the case without a sub-glycocalyx region. The size of the asymptotic regions have been exaggerated for illustrative purposes.

the small parameter $\delta$. The appropriate boundary and far-field conditions in Region I are

$$
\begin{array}{cl}
P=1 & \text { for } x \in \mathbb{R}, y=1, \\
\frac{\partial P}{\partial y}=0 & \text { for } x \in \mathbb{R}, y=0, \\
\nabla P \rightarrow 0 & \text { for }|x| \rightarrow \infty, y \in(0,1) .
\end{array}
$$

The system for Region I is defined by (13) in terms of the fluid pressure, from which the fluid velocity can be obtained, using the first two equations in (11). We may solve (13) using complexvariable theory introducing $z=x+i y$, with the transformation $\tanh \pi z / 4$ mapping the infinite-strip domain into a semicircle, with origin mapped to origin. Then the system (13) in transformed space satisfies the standard Green's function in an infinite two-dimensional domain, yielding the result

$$
P=1+\frac{\kappa}{\pi} \operatorname{Re}\left(\log \tanh \frac{\pi z}{4}\right),
$$

where $\operatorname{Re}(\cdot)$ denotes the real part. We show this Region I pressure and the streamlines it induces in Fig. 6. Additionally, we highlight that the far-field pressure is

$$
P \sim 1-\frac{2 \kappa}{\pi} e^{-\pi|x| / 2} \cos \frac{\pi y}{2} \quad \text { as }|x| \rightarrow \infty, y \in(0,1),
$$

which we note decays exponentially away from the intercellular cleft, justifying our restriction of the model to a single intercellular cleft.

For later matching purposes, it will be useful to note from (14) that

$$
P \sim \frac{\kappa}{\pi} \log |x|+1-\frac{\kappa}{\pi} \log \frac{4}{\pi} \quad \text { as }|x| \rightarrow 0 .
$$

We note that the Region I pressure appears to be logarithmically unbounded as we approach the intercellular cleft. This unphysical result is remedied in the next section, where we consider the problem in Region II, the transition region near the intercellular cleft entrance. Moreover, 

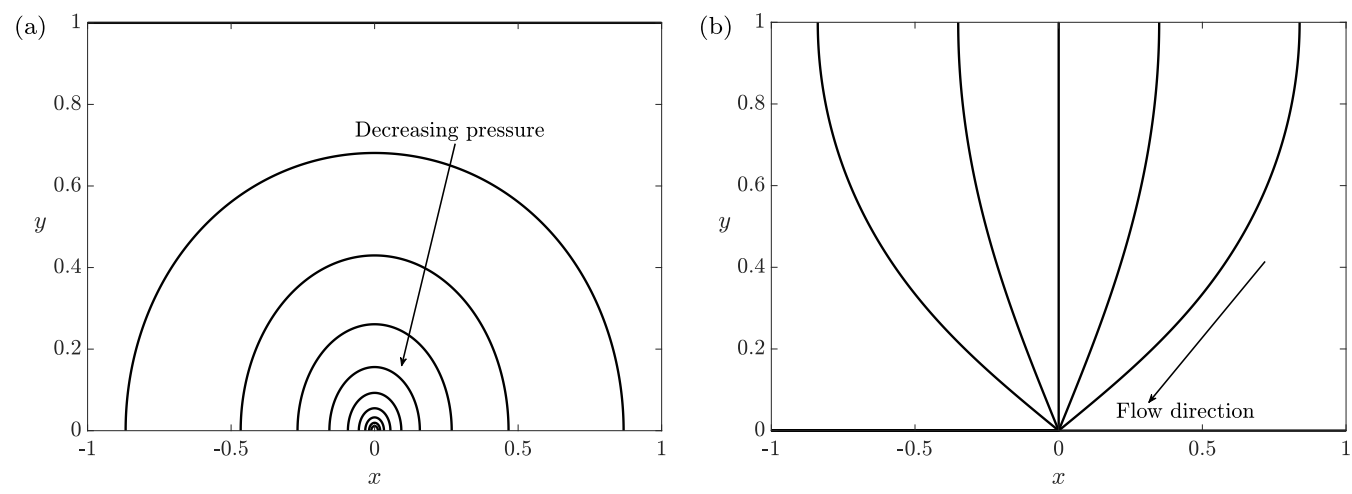

FIG. 6. (a) A pressure contour map in Region I from $P=0.55$ to $P=1$ in increments of 0.05 , as given by (14). (b) The streamlines in Region I, as given by the complex conjugate of (14). We use $\kappa=0.3$ for both panels.

investigating Regions II and III in turn will allow us to determine the value of $\kappa$ (currently unknown) in terms of the system parameters.

\section{Region II}

We scale into Region II using $\boldsymbol{x}=\delta \boldsymbol{X}=\delta(X, Y), \boldsymbol{U}=\overline{\boldsymbol{U}} / \delta=(\bar{U}, \bar{V}) / \delta$, and $u=\bar{u} / \delta$. Additionally, to capture all the relevant terms at once, we shall treat, in a well-established fashion, logarithmically large terms as 1 .

In the glycocalyx (Region IIa), the pressure satisfies Laplace's equation in the half-plane

$$
\nabla^{2} P=0 \quad \text { for } X \in \mathbb{R}, \quad Y \in \mathbb{R}^{+},
$$

with the flow given by $\bar{U}=-\nabla P$. In the intercellular cleft (Region IIb), the governing equations become

$$
p_{X}=\delta \xi \nabla^{2} \bar{u}, \quad p_{Y}=\delta \xi \nabla^{2} v, \quad \bar{u}_{X}+v_{Y}=0 \quad \text { for }|X|<1, Y<0 .
$$

The boundary and coupling conditions for this system arise from (12b)-(12d) and are

$$
\begin{aligned}
v & =\bar{V}, \quad p=P, \quad \bar{U}-\bar{u}=\alpha \frac{\partial \bar{u}}{\partial Y} \quad \text { for }|X|<1, \quad Y=0, \\
\frac{\partial P}{\partial Y} & =0 \quad \text { for }|X|>1, \quad Y=0, \\
\bar{u} & =v=0 \quad \text { for }|X|=1, \quad Y<0 .
\end{aligned}
$$

In the limit of $\delta \rightarrow 0^{+}$, we see from (17b) that the leading-order pressure within Region IIb is constant, and thus

$$
p=B,
$$

where $B$ is a constant to be determined via matching. In Region IIb, we note that the leading-order flow is coupled to the first-correction to the pressure, which is of $O(\delta)$. We do not need to solve this problem to obtain the fluid flux results in which we are interested; for an example of a related problem coupled in a similar manner where the inner flow problem must be solved to obtain the required outer results, the interested reader is directed to Ref. [37]. For the problem at hand, it suffices to note that the flux is conserved through Region II, and thus the flux entering Region IIa from Region I is equal to the flux entering Region III from Region IIb. This corresponds to the 
mathematical statement

$$
\lim _{r \rightarrow \infty} \int_{0}^{\pi} \frac{\partial P}{\partial r} r d \theta=\lim _{Y \rightarrow-\infty} \int_{-1}^{1}(-v) d X,
$$

which follows on application of the divergence theorem to the continuity equations in $(17 \mathrm{~b})$, and where $(r, \theta)$ are polar coordinates centered around $(X, Y)=(0,0)$. That the exact details of the flow in Region IIb have no bearing on the leading-order flux through the glycocalyx system immediately implies that the exact value of the Beavers-Joseph slip coefficient will have no leading-order effect on the flux through the system when there is no sub-glycocalyx.

Using (19), we note that the second equation in (18a) becomes

$$
P=B \text { for }|X|<1, Y=0 .
$$

Hence, the system for $P$, consisting of (17a), (18b), and (21), decouples from the remaining dependent variables. We solve this system using complex-variable theory in $Z=X+i Y$, noting that the transformation $\cosh ^{-1} Z$ maps the half-plane-with-a-slot $Z$ domain into a semi-infinite rectangle, and that the logarithmic far-field matching becomes linear in the transformed domain. Therefore, the system (17a), (18b), and (21) is solved by

$$
P=B+\frac{D}{\pi} \operatorname{Re}\left(\cosh ^{-1} Z\right),
$$

where $D$ is a constant to be determined via matching. For the purposes of matching, we note that the far-field behavior of (22) is

$$
P \sim \frac{D}{\pi} \log |Z|+\frac{D}{\pi} \log 2+B \quad \text { as }|Z| \rightarrow \infty,
$$

justifying the Dirac delta function in (13). Matching between Regions I and II using (16) and (23) with van Dyke's matching rule [38], we deduce that

$$
D=\kappa, \quad B=1-\frac{\kappa}{\pi} \log \frac{8}{\pi \delta} .
$$

Thus we have deduced the relevant terms in Region II in terms of $\kappa$, which is still to be determined. Using (20), the conservation of flux condition, we note that the dimensionless flux entering Region III from Region IIb can be written as

$$
\lim _{Y \rightarrow-\infty} \int_{-1}^{1}(-v) d X=\kappa,
$$

and we show the Region IIa pressure and streamlines in Fig. 7.

From (22) and (24), we may also determine that the flow velocity on the interface between glycocalyx and intercellular cleft is

$$
\bar{U}=0, \quad \bar{V}=\frac{\kappa}{\pi} \frac{1}{\sqrt{1-X^{2}}} \quad \text { for }|X|<1, Y=0 .
$$

There are integrable singularities in $(26)$ at $(|X|, Y)=(1,0)$, which are artifacts that arise due to the sharp corners in our model geometry. Our remaining task is to couple the analysis thus far with the flow in the intercellular cleft, in Region III, which will allow us to close the system and determine $\kappa$, the sole remaining free parameter, in terms of the system parameters.

\section{Region III}

In Region III we use $Y=\hat{Y} / \delta$ and $\bar{u}=\delta \hat{u}$ to scale the Stokes equations (17b) and obtain the following at leading order:

$$
p_{X}=O\left(\delta^{2}\right), \quad p_{\hat{Y}}=\xi v_{X X}+O\left(\delta^{2}\right), \quad \hat{u}_{X}+v_{\hat{Y}}=0 \quad \text { for }|X|<1, \quad \hat{Y} \in(-\lambda, 0) .
$$



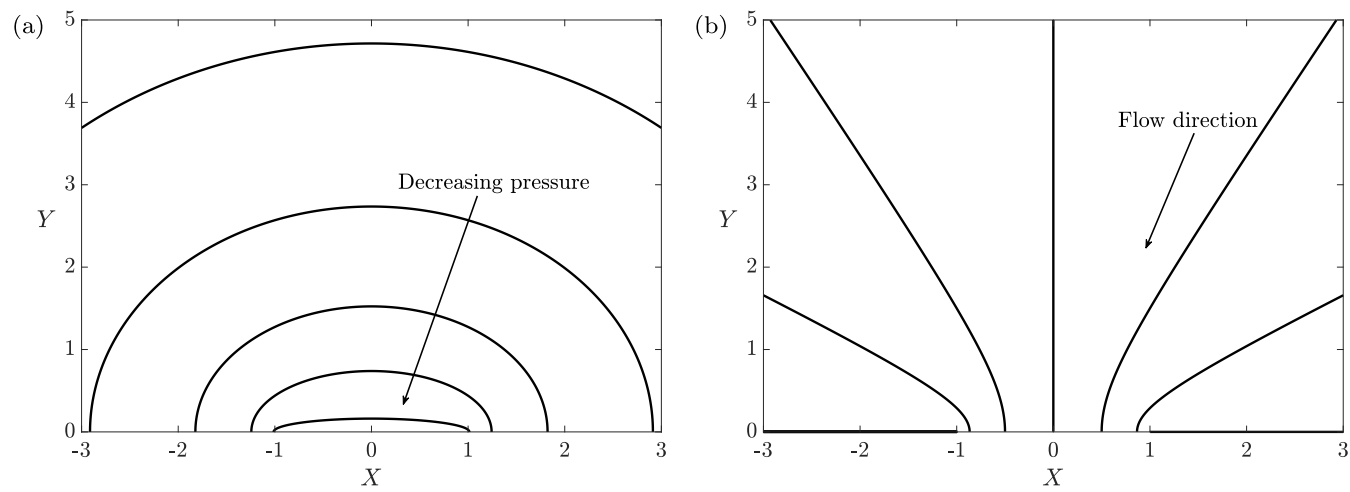

FIG. 7. (a) A pressure contour map in Region IIa from $P=0.63$ to $P=0.83$ in increments of 0.05 , as given by (22) and (24). (b) The streamlines in Region IIa, as given by (22) and (24). We use $\kappa=0.3$ and $\delta=0.05$ in both figures.

The appropriate boundary conditions are (12d) and (12e), which become

$$
\begin{aligned}
& \hat{u}=v=0 \quad \text { for }|X|=1, \quad \hat{Y} \in(-\lambda, 0), \\
& p=0 \quad \text { for }|X|<1, \quad \hat{Y}=-\lambda .
\end{aligned}
$$

The system (27) is solved by

$$
\hat{u}=0, \quad v=C \frac{X^{2}-1}{2 \xi}, \quad p=C(\hat{Y}+\lambda),
$$

where $C$ is a constant to be determined by one last asymptotic matching procedure between Regions IIb and III.

To match these regions, we first note from (19) and (24) that $p$ is constant at leading order within Region II. Thus, we are able to deduce that

$$
C=\frac{1}{\lambda}-\frac{\kappa}{\lambda \pi} \log \frac{8}{\pi \delta} .
$$

Second, we note from the continuity equation in (27a) that the flux in Region III is conserved. As the flux into Region III is given by (25), we may deduce that

$$
\kappa=-\int_{-1}^{1} v(X,-\lambda) d X=\frac{2 C}{3 \xi},
$$

and hence

$$
\kappa=\frac{2}{3 \xi \lambda+\frac{2}{\pi} \log \frac{8}{\pi \delta}},
$$

thus completing the asymptotic solution for Case 1 .

We can calculate the dimensional flux through the system, defined in (6), using (10), (28), (29), and (31), to obtain

$$
Q=\frac{\Pi}{\mu} \frac{d^{3}}{12 L+\frac{d^{3}}{\pi k} \log \frac{16 H}{\pi d}},
$$

leading to an effective resistance, defined in (7), of

$$
K=\frac{12 L}{d^{3}}+\frac{1}{\pi k} \log \frac{16 H}{\pi d} .
$$




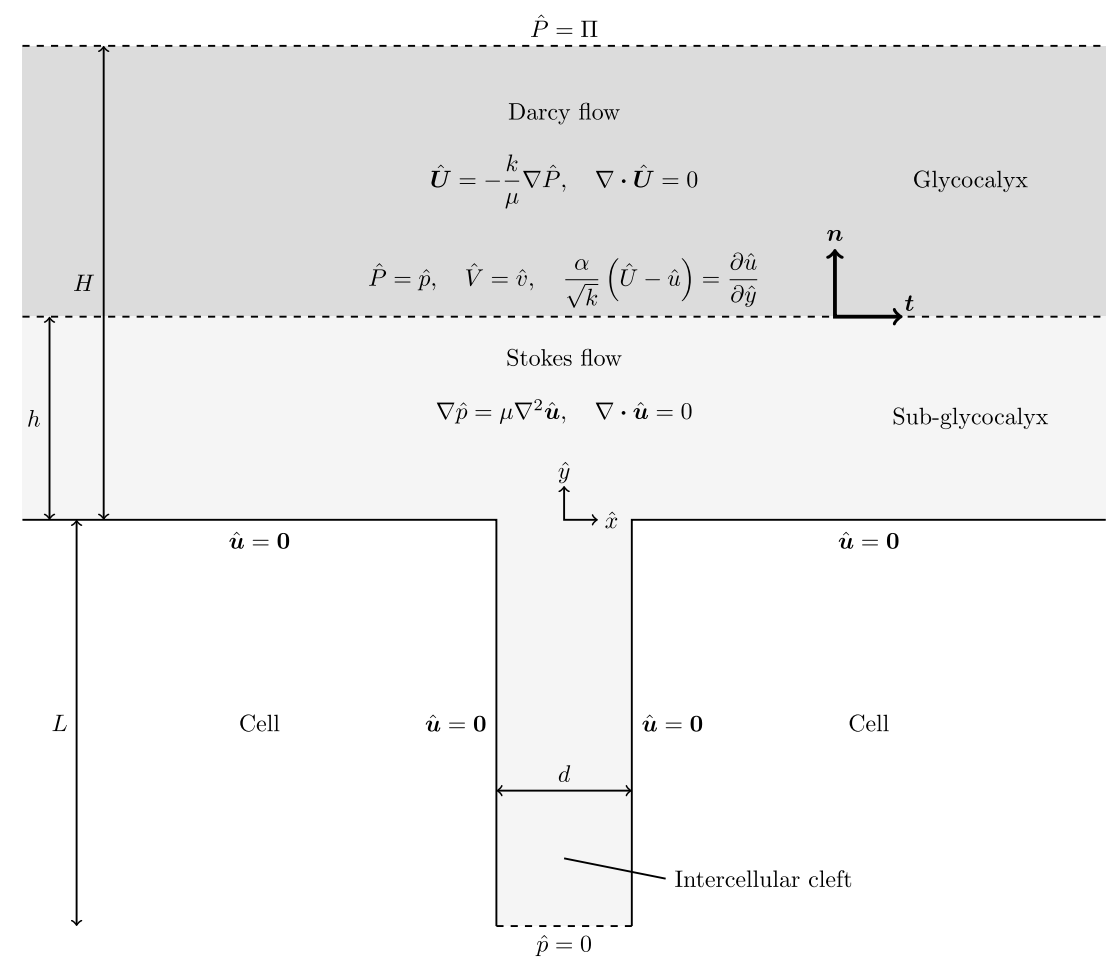

FIG. 8. Schematic of the dimensional flow problem with a sub-glycocalyx. We consider a two-dimensional setup, with the impermeable sub-glycocalyx and intercellular cleft walls representing the endothelial cells. Darcy flow governs the dark gray region, Stokes flow governs the light gray region, and the white regions denote the impermeable endothelial cells.

The first term on the right-hand side of (33) is due to the intercellular cleft and the second is due to the structure of the glycocalyx. Therefore it is the latter that will be useful in comparing the effective resistance in this case to that in the second case, for which there is a significant sub-glycocalyx space $(h>0)$. We do this in the next section, where we show that the dimensional flux and effective resistance have different dependencies on the system parameters. Additionally, we will also show that the model we present in the next section becomes invalid when $h \rightarrow 0$, and that this limit is when Case 1, the model we have considered in this section, is valid.

\section{CASE 2: SIGNIFICANT SUB-GLYCOCALYX SPACE}

We now consider the problem with a sub-glycocalyx space, corresponding to $0<h<H$. With the addition of the no-slip condition

$$
\boldsymbol{u}=\mathbf{0} \text { for }|\hat{x}|>d / 2, \hat{y}=0,
$$

at the boundary where the sub-glycocalyx space meets the endothelial cells, the governing system for Case 2 is defined by (1)-(5) and (34). Additionally, we note that $\Gamma$, the interface for (3), is now defined as

$$
\Gamma=\{(\hat{x}, \hat{y}): \hat{x} \in \mathbb{R}, \hat{y}=h\} .
$$

We give a schematic of our model setup and the governing equations in Fig. 8. 
We will solve the system (1)-(5) and (34) for $h>0$ by exploiting the extreme parameter ratios discussed in Sec. II. To highlight the important parameters in the system and facilitate an asymptotic analysis, we now nondimensionalize the system.

\section{A. Dimensionless problem}

To obtain a distinguished asymptotic limit, we use the following lubrication-type scalings:

$$
(\hat{U}, \hat{V}, \hat{u}, \hat{v})=\frac{k \Pi}{\mu H}(\epsilon U, V, u / \epsilon, v), \quad(\hat{P}, \hat{p})=\Pi(P, p), \quad(\hat{x}, \hat{y})=l(x, \epsilon y),
$$

where we preemptively define the horizontal lengthscale as

$$
l=\left[\frac{h^{3}(H-h)\left(a h+4 k^{1 / 2}\right)}{12 k\left(a h+k^{1 / 2}\right)}\right]^{1 / 2},
$$

which describes the typical horizontal extent of flow in the glycocalyx, and we define the dimensionless aspect ratio as $\epsilon=H / l$. In (37), the $a h$ and $k^{1 / 2}$ terms arise due to the Beavers-Joseph interfacial condition, and the remaining terms arise due to the geometry of the glycocalyx.

Using the scalings (36), we obtain the following dimensionless governing equations in the glycocalyx:

$$
U=-P_{x}, \quad V=-P_{y}, \quad \epsilon^{2} U_{x}+V_{y}=0,
$$

and in the sub-glycocalyx and intercellular cleft

$$
p_{x}=\beta\left(u_{y y}+\epsilon^{2} u_{x x}\right), \quad p_{y}=\epsilon^{2} \beta\left(v_{y y}+\epsilon^{2} v_{x x}\right), \quad u_{x}+v_{y}=0 .
$$

Here we define the dimensionless parameters $\eta=h / H, \bar{\alpha}=k^{1 / 2} / a H$, and $\beta=k l^{2} / H^{4}$, and, for clarity, we use the following domain descriptions:

$$
\begin{aligned}
\text { Glycocalyx } & =\{(x, y): x \in \mathbb{R}, y \in(\eta, 1)\}, \\
\text { Sub-glycocalyx } & =\{(x, y): x \in \mathbb{R}, y \in(0, \eta)\}, \\
\text { Intercellular cleft } & =\{(x, y): x \in(-\bar{\delta}, \bar{\delta}), y \in(-v / \epsilon, 0)\},
\end{aligned}
$$

where we additionally define the dimensionless parameters $\bar{\delta}=d / 2 l$ and $v=L / l$. We note that $\beta$ can be written in terms of $\eta$ and $\bar{\alpha}$, but, to avoid cumbersome expressions in the dimensionless system for Case 2, we retain its use. We will discuss the asymptotic sizes of our dimensionless parameters in Sec. IV B. Finally, the dimensionless boundary and coupling conditions are

$$
\begin{aligned}
& P=1 \quad \text { for } x \in \mathbb{R}, \quad y=1, \\
& v=V, \quad p=P, \quad u-\epsilon^{2} U=-\bar{\alpha} \frac{\partial u}{\partial y} \quad \text { for } x \in \mathbb{R}, y=\eta, \\
& \boldsymbol{u}=\mathbf{0} \quad \text { for }|x|>\bar{\delta}, \quad y=0, \\
& \boldsymbol{u}=\mathbf{0} \quad \text { for }|x|=\bar{\delta}, \quad y \in(-v / \epsilon, 0), \\
& p=0 \quad \text { for }|x|<\bar{\delta}, \quad y=-v / \epsilon .
\end{aligned}
$$

\section{B. Asymptotic structure}

We consider the asymptotic limits of $H / l \ll 1$ and $d / L \ll 1$, with the remaining dimensionless parameters being of $O(1)$. These asymptotic limits correspond to lubrication flow in the subglycocalyx layer and in the intercellular cleft, respectively. The first limit follows from $k \ll h^{2}$, which is required for the Stokes region to be distinguishable from the Darcy region (as discussed in Sec. II), and the second limit follows from having a long, thin intercellular cleft; in terms 


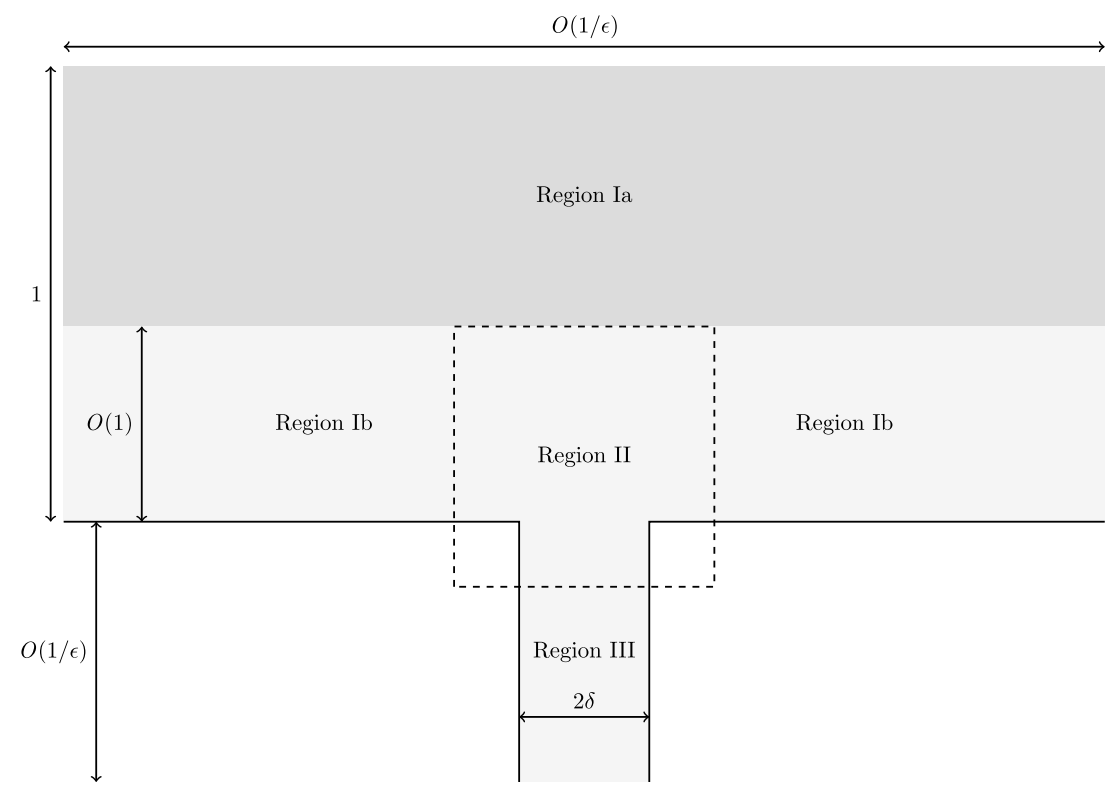

FIG. 9. The asymptotic structure of the problem in the case with a sub-glycocalyx region. For visual clarity, the asymptotic regions are not to scale.

of dimensionless parameters, these asymptotic limits are $\epsilon \rightarrow 0^{+}$and $\bar{\delta} \rightarrow 0^{+}$. We proceed by performing an asymptotic analysis in these two small parameters. With regard to $\eta=O(1)$, we note from (37) that $\epsilon=O\left(k^{1 / 2} / h \eta^{1 / 2}\right)$ as $h \rightarrow 0$. As $k^{1 / 2} / h \ll 1$, and we must have $\epsilon \ll 1$ for the asymptotic analysis that follows to be valid, we see that our asymptotic limit will break down when $\eta$ is small-specifically, when $\eta=O\left(k / h^{2}\right)$, which corresponds to $h=O\left((k H)^{1 / 3}\right)$ (approximately $h=5 \mathrm{~nm}$ using Table I). To summarize, if $\eta=O(1)$ then the lubrication scalings in this section are valid; however, as $\eta$ becomes small the horizontal extent of the flow becomes of $O(H)$ and our analysis in Sec. III is valid. Finally, although the value of $a$, the Beavers-Joseph slip coefficient, is unknown, we take the dimensionless $\bar{\alpha}$ to be of $O(1)$ because this results in a distinguished asymptotic limit from the tangential slip condition, and will allow us to quantify the influence of the parameter $a$ fully.

We show the asymptotic structure of the problem in Fig. 9. At leading order in Region I, the flow is vertical in the glycocalyx (Region Ia) but horizontal in the sub-glycocalyx (Region Ib). As the leading-order flow in the intercellular cleft is also vertical, the transition from horizontal to vertical occurs close to the intercellular cleft entrance, which we term Region II. In this region, the effect of flow from Region Ia is not felt at leading order. Finally, Region II transitions into Region III, the main intercellular cleft region. Here the leading-order flow is vertical.

\section{Solving for the flow}

In the glycocalyx (Region Ia) and sub-glycocalyx (Region Ib), the leading-order system is obtained by taking the limit $\epsilon \rightarrow 0^{+}$in (38). In Region Ia, the leading-order equations are

$$
U=-P_{x}, \quad V=-P_{y}, \quad V_{y}=0 \quad \text { for } x \in \mathbb{R}, \quad y \in(\eta, 1),
$$

and in Region Ib they are

$$
p_{x}=\beta u_{y y}, \quad p_{y}=0, \quad u_{x}+v_{y}=0 \quad \text { for } x \in \mathbb{R}, \quad y \in(0, \eta) .
$$


The relevant boundary conditions for Region I are the leading-order versions of (40a)-(40c):

$$
\begin{aligned}
& P=1 \quad \text { for } x \in \mathbb{R}, \quad y=1, \\
& v=V, \quad p=P, \quad u=-\bar{\alpha} \frac{\partial u}{\partial y} \quad \text { for } x \in \mathbb{R}, \quad y=\eta, \\
& u=0, \quad v=0 \quad \text { for } x \in \mathbb{R}, \quad y=0 .
\end{aligned}
$$

To solve this system we first determine the flow in the sub-glycocalyx in terms of $p$, then we use the coupling conditions to solve for the flow in the glycocalyx, also in terms of $p$, which will then enable us to obtain a closed governing equation for $p$. This will also require an asymptotic matching procedure with Regions II and III (carried out in the Appendix) to close the system.

We solve (41b) with the tangential slip condition in (42b) and the no-slip condition (42c) to obtain

$$
u=\frac{1}{2 \beta}\left(y^{2}-\eta \frac{\eta+2 \bar{\alpha}}{\eta+\bar{\alpha}} y\right) \frac{d p}{d x}, \quad v=\frac{1}{12 \beta}\left(3 \eta \frac{\eta+2 \bar{\alpha}}{\eta+\bar{\alpha}} y^{2}-2 y^{3}\right) \frac{d^{2} p}{d x^{2}},
$$

noting that $p=p(x)$, which will be determined from (45) below. Then, using (41a), (42a), and the continuity of flux condition in (42b), we deduce

$$
U=\frac{y-1}{1-\eta} \frac{d^{3} p}{d x^{3}}, \quad V=\frac{1}{1-\eta} \frac{d^{2} p}{d x^{2}}, \quad P=1+\frac{1-y}{1-\eta} \frac{d^{2} p}{d x^{2}},
$$

where the reduction of the right-hand side for $V$ follows from the definitions of $l$ and $\beta$ in Sec. IV A. Then, imposing the continuity of pressure condition in (42b), we obtain the governing equation

$$
\frac{d^{2} p}{d x^{2}}-p=-1+[2 \gamma p(0,0)] \hat{\delta}(x, y)
$$

where $\hat{\delta}(x, y)$ is a Dirac delta function which appears from matching with Regions II and III (performed in the Appendix), and

$$
\gamma=\frac{d^{3}}{4 h L}\left[\frac{(H-h)\left(a h+k^{1 / 2}\right)}{3 k h\left(a h+4 k^{1 / 2}\right)}\right]^{1 / 2} .
$$

In (45), the right-hand side represents the pressure forcing in the system, from just above the glycocalyx to the entrance of the intercellular channel. In particular, the Dirac delta function in (45) arises from continuity of flux across Region II, from which we are also able to determine $Q$, the dimensional fluid flux through the system, in terms of the pressure at the cleft entrance, given by

$$
Q=\left.\left(\frac{\Pi d^{3}}{12 \mu L}\right) p\right|_{x=\mathbf{0}}
$$

The solution to (45) is

$$
p=1-\frac{\gamma}{1+\gamma} e^{-|x|},
$$

and we illustrate $p(x)$ for different values of $\gamma$ in Fig. 10. We note that the pressure (and thus the flow) decays exponentially away from the intercellular cleft, justifying our restriction of the model to a single intercellular cleft. The nonanalyticity in (48) arises from the intercellular cleft appearing as a point sink in this region. It could be smoothed out by solving for the pressure within Region II in Appendix A 1, but we restrict that analysis to deriving the information required to close the system. Using (46) and (48) in (47), we may calculate the dimensional flux through the system in terms of the system parameters, given by

$$
Q=\frac{\Pi}{\mu} \frac{d^{3}}{12 L+d^{3}\left[\frac{3(H-h)\left(a h+k^{1 / 2}\right)}{k h^{3}\left(a h+4 k^{1 / 2}\right)}\right]^{1 / 2}} .
$$




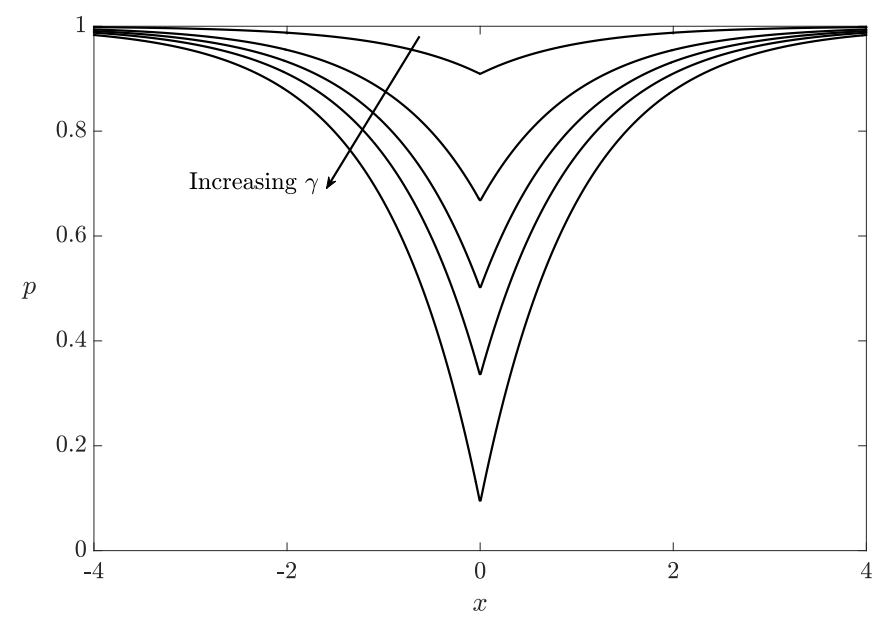
in (48).

FIG. 10. The pressure distribution within the sub-glycocalyx region for $\gamma=0.1,0.5,1,2,10$, as defined

We can then use (49) to determine the effective resistance, defined in (7), as

$$
K=\frac{12 L}{d^{3}}+\left[\frac{3(H-h)\left(a h+k^{1 / 2}\right)}{k h^{3}\left(a h+4 k^{1 / 2}\right)}\right]^{1 / 2} .
$$

In a similar manner to (33), the first term on the right-hand side of (50) is due to the intercellular cleft, and the second to the structure of the glycocalyx. Therefore, the second is the more useful for comparing the effective resistance between cases.

\section{COMPARISON BETWEEN CASES}

Our asymptotic results allow us to determine the key differences between the flow structures in each case. In this section we restate and summarize these results for convenience and compare them. We summarize the key quantitative differences in Table II. Recall that parameter definitions are given in Sec. II.

In Case 1, where the sub-glycocalyx space is negligible, the horizontal extent of the flow within the glycocalyx is of $O(H)$, the same order as the height of the glycocalyx. The horizontal extent of the flow is much larger when the sub-glycocalyx space is significant (Case 2), where it is of $O(l)$, given by

$$
l=\left[\frac{h^{3}(H-h)\left(a h+4 k^{1 / 2}\right)}{12 k\left(a h+k^{1 / 2}\right)}\right]^{1 / 2},
$$

TABLE II. A comparison of the differences between the two cases we consider in this paper.

\begin{tabular}{lcc}
\hline \hline & Case 1 & Case 2 \\
\hline Horizontal extent of flow in eGlx & $O(H)$ & $O(l)$ \\
Magnitude of vertical flow in eGlx & $O\left(\frac{k \Pi}{\mu d}\right)$ & $O\left(\frac{k \Pi}{\mu H}\right)$ \\
Magnitude of horizontal flow in eGlx & $O\left(\frac{k \Pi}{\mu H}\right)$ & $O\left(\frac{k \Pi}{\mu l}\right)$ \\
Magnitude of vertical flow in sub-glycocalyx space & $\mathrm{N} / \mathrm{A}$ & $O\left(\frac{k \Pi}{\mu H}\right)$ \\
Magnitude of horizontal flow in sub-glycocalyx space & $\mathrm{N} / \mathrm{A}$ & $O\left(\frac{k \Pi l}{\mu H^{2}}\right)$ \\
Effective resistance due to glycocalyx structure & $\frac{1}{\pi k} \log \frac{16 H}{\pi d}$ & $\sqrt{\frac{3(H-h)\left(a h+k^{1 / 2}\right)}{k h^{3}\left(a h+4 k^{1 / 2}\right)}}$ \\
\hline \hline
\end{tabular}




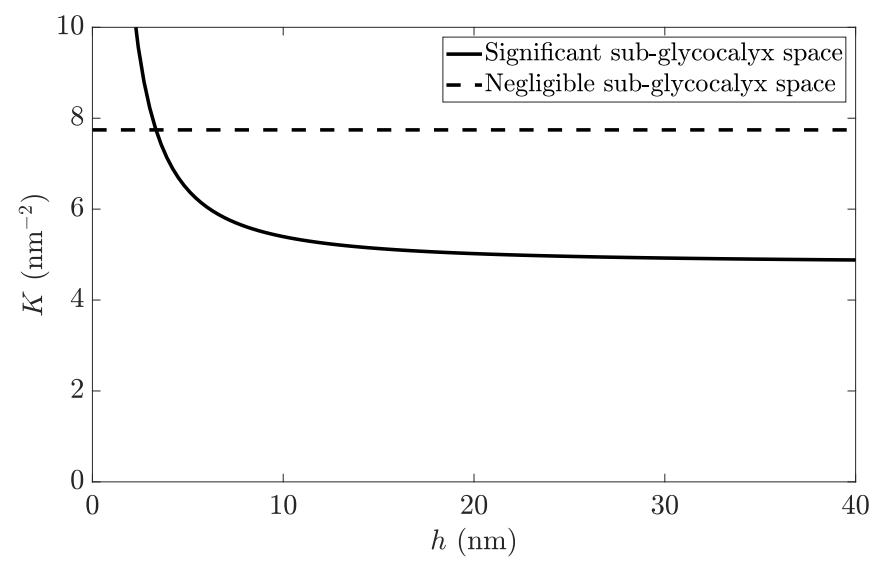

FIG. 11. The effective resistance for the two cases we have considered in this paper, with and without a sub-glycocalyx space, given in (52). As discussed in this paper, the case with a sub-glycocalyx space is valid for larger values of $h$, and the case with a negligible sub-glycocalyx space becomes valid when $h=O\left([k H]^{1 / 3}\right) \approx$ $O(5 \mathrm{~nm})$, which is very close to the intersection point between the two lines. We use the parameter values in Table I, with variable $h$. We also use $a=0.025$, corresponding to $a=(2 k)^{1 / 2} / h$ if we were to fix $h=40 \mathrm{~nm}$.

originally given in (37). Using (51), we note that $l \gg H$ is self-consistent with our observation in Sec. IV B that we require $h^{3} \gg H k$ for Case 2 to be valid. Therefore, our results suggest that a significant sub-glycocalyx gap causes a greater horizontal extent of the glycocalyx to be used for filtration (which is advection-dominated). If the sub-glycocalyx gap is negligible, only the parts of the glycocalyx near each intercellular cleft will be used for filtration.

We may also use our asymptotic results (summarized in Table II) to obtain the magnitude of the flow within the glycocalyx in each case. In Case 1, the horizontal velocity in the main part of the glycocalyx has magnitude of $O(k \Pi /[\mu H])$, and the vertical velocity has magnitude of $O(k \Pi /[\mu d])$. Therefore, the magnitude of the vertical velocity is significantly higher than that of the horizontal, and the streamlines are vertical to leading order within the glycocalyx. In Case 2, the horizontal velocity in the main part of the glycocalyx has magnitude of $O(k \Pi /[\mu l])$, and the vertical velocity has magnitude of $O(k \Pi /[\mu H])$. Therefore, the streamlines are again vertical to leading order within the glycocalyx here, but the strength of the flow within the glycocalyx is significantly smaller than in Case 1. This is because the horizontal extent of the flow is far greater. Within the sub-glycocalyx region in Case 2, the horizontal velocity has magnitude of $O\left(k \Pi l /\left[\mu H^{2}\right]\right)$, and the vertical velocity has magnitude of $O(k \Pi /[\mu H])$. Here the streamlines are horizontal to leading order, as fluid from a large horizontal extent must be transported to the intercellular cleft in the center of the domain.

It is also helpful to compare the effective resistances in each case, given by

$$
\begin{aligned}
& K=\frac{12 L}{d^{3}}+\frac{1}{\pi k} \log \frac{16 H}{\pi d} \text { in Case } 1, \\
& K=\frac{12 L}{d^{3}}+\left[\frac{3(H-h)\left(a h+k^{1 / 2}\right)}{k h^{3}\left(a h+4 k^{1 / 2}\right)}\right]^{1 / 2} \text { in Case 2, }
\end{aligned}
$$

originally given in (33) and (50), respectively. In Fig. 11 we compare the two resistances in (52) graphically. In both expressions, the first term on the right-hand side is the resistance due to the intercellular cleft and the second term on the right-hand side is the resistance due to the glycocalyx structure. The appropriate comparison is therefore between the second term on the right-hand sides of the effective resistances.

In particular, we note that the effective resistance in Case 2 decreases as $h$ increases (Fig. 11); the presence of a sub-glycocalyx results in a lower effective resistance through the system, and this 
allows for a greater fluid flux through the glycocalyx structure for a given pressure difference, as one might expect on physical grounds. Although a naive inspection of (52b) would suggest that $K$ scales with $h^{-3 / 2}$ as $h \rightarrow 0$, our Case 1 analysis tells us that this is not the case: in fact, the effective resistance from Case 2 is bounded as $h$ is reduced, as seen from (52a), and Case 1 becomes a more appropriate model when $h=O\left([k H]^{1 / 3}\right)$.

We also note that the effective resistance has an inversely proportional relationship with the effective permeability $k$ for Case 1 , but an inverse square-root relationship for Case 2 . Thus, the permeability of the glycocalyx is more important when there is a negligible sub-glycocalyx space. Perhaps less intuitively, we note that the effective resistance has a logarithmic relationship with the height of the glycocalyx $H$ for Case 1, but a square-root relationship for Case 2. Thus, a thicker glycocalyx increases the effective resistance (with diminishing returns) in both cases, but this effect is more pronounced in Case 2.

Finally, we note that the Beavers-Joseph slip coefficient $a$ does not contribute to the leading-order effective resistance in Case 1. However, in Case 2 we see that although the effective resistance increases as $a$ increases, the effect of varying $a$ is fairly small. That is, as $a$ varies from 0 to $\infty$, the second term on the right-hand side of (52b) increases by only a factor of two. This is significantly less than the effect of the other system parameters.

\section{DISCUSSION}

We investigated the flow through the glycocalyx for two plausible structures of the glycocalyx: the first with a negligible sub-glycocalyx space, the second with a significant sub-glycocalyx space. In the analysis of both cases, we exploited the small aspect ratio in the geometry of the problem to investigate the system via an asymptotic reduction. Our asymptotic analysis allowed us to derive analytic results for the flow through the glycocalyx in terms of the system parameters. We found that when the sub-glycocalyx space was significant, the horizontal extent of the flow was far greater, providing an estimate of the proportion used for filtration. Moreover, the presence of a sub-glycocalyx gap provided less overall resistance for the plasma flow through the system, allowing for a greater flux for a given pressure drop. We summarize the key differences between the two cases in Sec. V. Our theoretical predictions provide a way to estimate some microscale properties of the glycocalyx, such as the permeability, that cannot be calculated using current imaging technology.

We note that the flux we calculate in this paper, $Q$, has units of area per time. To obtain the fluid filtration flux across the capillary wall per unit area used in the revised Starling hypothesis, which has units of length per time, we can multiply $Q / 2$ by the typical perimeter of an endothelial cell and divide this by the typical area of an endothelial cell, both as viewed on the surface of the capillary wall. We use the factor of $1 / 2$ to counter the double counting of adjacent cells. Using Table I for typical parameter values, and assuming that an endothelial cell is approximately circular on the surface of a capillary wall, this yields a fluid filtration flux across the capillary wall per unit area of approximately $2 Q / D=2 \Pi / \mu K D=0.04-0.2 \mu \mathrm{m} \mathrm{s}^{-1}$, where the lower bound of our estimate corresponds to the case where the height of the sub-glycocalyx space is negligible. Here, $D$ is the typical "diameter" of the cell as seen on the surface of the capillary wall, $\Pi$ is the difference in hydraulic pressure across the glycocalyx system, $\mu$ is the viscosity of the interstitial fluid, and $K$ is the effective resistance, defined in (33) and (50) for the cases where the height of the sub-glycocalyx space is negligible and significant, respectively. Although our approximation shows reasonable agreement with measured fluid filtration fluxes [26], it does slightly overestimate the fluid filtration flux. We attribute this overestimation to the fact that our model does not take any solute transport into account: both the osmotic pressure across the glycocalyx and an increase in the effective plasma viscosity due to solute transport would cause lower values of the fluid filtration flux. We also note that, in a similar manner to the above, we are able to estimate the local hydraulic conductivity present in Starling's hypothesis by $2 / \mu K D$. This provides a way to link the microscale properties of the glycocalyx with the macroscale properties used in Starling's hypothesis. Additionally, although we do not consider the solute transport problem here, it would be possible to model the effect of an 
oncotic pressure by modifying the effective pressure difference across the endothelial glycocalyx layer. As the pressure difference is a multiplicative scaling of the variables in the problem, this would not qualitatively change any flow properties.

The two parameters of which we have the most uncertainty are the Beavers-Joseph slip coefficient and the height of the sub-glycocalyx layer, with no information regarding the former. As our model predicts the effective resistance and flux through the system, we could use these results to predict these two parameters by data fitting with appropriate experiments. However, we do note that our model suggests that the exact value of the Beavers-Joseph slip coefficient is unimportant to the effective resistance and flux through the system when the height of the sub-glycocalyx space is negligible.

With regard to the sub-glycocalyx layer, our analysis suggests several experiments that could be carried out to determine the extent of this space. As noted above, one of the key differences between the two cases is the horizontal extent of the flow within the glycocalyx structure. Therefore, it would be possible to estimate the extent of the sub-glycocalyx space if it were possible to track streamlines of the flow. This would ideally be carried out in real time, although this may be too delicate to carry out with current technology. An alternative method could be to track the distribution of nonbinding large molecules being sieved through the glycocalyx structure, for example organic nanodots [39]. If the majority of the molecules were deposited in very localized horizontal regions, this would provide evidence for a negligible sub-glycocalyx space. However, if the large molecules were deposited more uniformly in the horizontal direction, this would provide evidence for a significant sub-glycocalyx space, with the extent of deposition being related to the height of the sub-glycocalyx space. An additional experiment to determine the extent of the space takes advantage of our theoretical predictions that the effective resistance of the glycocalyx has a square-root dependence on the glycocalyx height when the sub-glycocalyx space is significant, but only a logarithmic dependence when the space is negligible. If systematic experiments could be carried out using the glycocalyx height as a variable parameter, perhaps by biochemical manipulation, this could shed light on the height and extent of the sub-glycocalyx space.

A theoretical understanding of how the flow through the endothelial glycocalyx layer is affected by a sub-glycocalyx space is an important first step towards understanding the size of such a space. Although we could model the effect of an oncotic pressure in the manner mentioned above, it is our hope that the hydrodynamical results we have derived in this paper will be formally combined with models for molecular transport, such as Ref. [40], and used to predict outcomes that cannot be directly obtained directly through experimental procedures.

In this paper we have neglected additional factors which might affect transport, such as electric effects generated by the active transport of ions across the cell membrane [41] and glycocalyx deformability [4]. While the framework we have introduced could be modified to account for these effects, it is also helpful to hypothesize on how these effects may change our results. If the electrokinetic effects led to flow reversal near the endothelial cells, as seen in Ref. [42], this would lead to an increase in the effective flow resistance. Although this effect would be more pronounced when the hydrostatic pressure difference across the endothelial glycocalyx layer was smaller, it is worth noting that a recent model for fluid transport across the retinal pigment epithelium (RPE) accounting for electrokinetic effects suggests that the electroosmotic effects were around three orders of magnitude smaller than osmotic effects for water transport [43]. Given that one would expect the net hydrostatic and osmotic pressure difference across the RPE to be lower than that across a vascular wall, the inclusion of electrokinetic effects is unlikely to have a significant effect on the effective flow resistance. However, the electrokinetic effects would be important for ion transport, and we would expect the negative endothelial glycocalyx fixed charge density to contribute directly to molecular transport by effectively changing the pore size, particularly for larger molecules such as albumin with a reflection coefficient close to 1 . With regard to glycocalyx deformability, while a increased external pressure may compress the glycocalyx slightly, decreasing its permeability and the size of any sub-glycocalyx space, it would also make the glycocalyx shorter, reducing the distance fluid has to travel. As these two mechanisms have competing effects on the 
filtration resistance, it is likely that the exact way in which glycocalyx compression affects its permeability would be important.

We have modeled the flow in the intercellular cleft using the lubrication equations. However, we note that if the cleft width were comparable to the typical pore size in the glycocalyx it would be possible to use the Darcy flow equations in the cleft region instead of the lubrication equations. Although it would not change the nature of our solutions (we would obtain the same results averaged over the cleft cross section), if the entire system were to be solved numerically it may be preferable to pose the Darcy flow equations instead of the lubrication equations when there is no sub-glycocalyx space (Case 1), since this avoids the issue of coupling different flow domains.

We also note that we have ignored the effect of any junction strands within the intercellular cleft for mathematical expediency. Although we expect this cleft to have an effect on the flow, we do not expect it to qualitatively affect the flow within either the glycocalyx or the sub-glycocalyx space. By changing the geometry of the problem we consider here, it would be possible to extend this work to consider the effect of a junction strand within the intercellular cleft as well as the effect of a sub-glycocalyx space. Moreover, our model does not fully account for the slow variation (compared to the "fast" variation associated with the distance between intercellular clefts) of pressure along a blood vessel over many endothelial cells. This is because we have assumed the pressure to be constant at the top of the glycocalyx. While this is likely to be correct at leading order, a more refined model could include the effect of blood flowing within the lumen above the glycocalyx, which would involve a slow variation of pressure along the top of the glycocalyx and the periodic distribution of many intercellular clefts. As this is a multiscale problem, one could use homogenization theory [44] to upscale this microscale problem systematically to derive a homogenized equation which describes the flux over the macroscale.

\section{ACKNOWLEDGMENTS}

This work was initiated at the Multi-scale Biology Study Group held at the University of Birmingham in December 2016, supported by the UK Multi-Scale Biology Network (MSB-Net) and the Predictive Modelling for Healthcare Technology through Maths Network (POEMS) funded by the Biotechnology and Biological Sciences Research Council and the Engineering and Physical Sciences Research Council, respectively. The authors would also like to thank the other participants of the Study Group. M.P.D. and J.R.K. were partly supported by the Biotechnology and Biological Sciences Research Council (Grant No. BB/L013940/1) and the Engineering and Physical Sciences Research Council, jointly funding the first grant number. K.A. would like to thank the British Heart Foundation (PG/15/37/31438), Bizkaia Talent Fellowship Scheme (AYD-000-256), and the Medical Research Council (MR/P003214/1) for salary support over the period of this research.

\section{APPENDIX: CLOSING THE SUB-GLYCOCALYX PROBLEM}

\section{Region II}

In this Appendix, we derive the closure conditions for Region Ib in the case with a sub-glycocalyx space. When $x=O(\bar{\delta})$ in Region Ib, the finite nature of the intercellular cleft becomes apparent. In this transition region, which we define as Region II (see Fig. 9), the vertical velocity grows in magnitude to accommodate the transition from horizontal to vertical flow within the intercellular cleft. Thus, we must additionally scale $v=O(1 / \bar{\delta})$. In this region, the relative sizes of $\epsilon$ and $\bar{\delta}$ become important. We consider the distinguished limit where $h=O(d)$, corresponding to $\epsilon \eta=$ $O(\bar{\delta})$, and thus we write $\epsilon \eta=\bar{\delta} \psi$, where $\psi=O(1)$. Writing $x=\bar{\delta} \bar{x}$ and $v=\bar{v} / \bar{\delta}$, and using the asymptotic expansions

$$
p \sim \frac{1}{1+\gamma}+\bar{\delta} p_{1}, \quad u \sim u_{0}, \quad \bar{v} \sim \bar{v}_{0}
$$


noting that $p$ is constant at leading order in this region, where $\gamma$ is defined in (46), we obtain the governing equations

$$
p_{1 \bar{x}}=\beta\left(u_{0 y y}+\frac{\psi^{2}}{\eta^{2}} u_{0 \bar{x} \bar{x}}\right), \quad p_{1 y}=\frac{\psi^{2} \beta}{\eta^{2}}\left(\bar{v}_{0 y y}+\frac{\psi^{2}}{\eta^{2}} \bar{v}_{0 \bar{x} \bar{x}}\right), \quad u_{0 \bar{x}}+\bar{v}_{0 y}=0 .
$$

The flux through this region is conserved, and thus

$$
\lim _{\bar{x} \rightarrow \infty} \int_{0}^{1} u(\bar{x}, y) d y-\lim _{\bar{x} \rightarrow-\infty} \int_{0}^{1} u(\bar{x}, y) d y=\lim _{y \rightarrow-\infty} \int_{-1}^{1} \bar{v}(\bar{x}, y) d \bar{x},
$$

where there is no contribution from Region Ia because of the asymptotic increase from $v$ to $\bar{v}$. As we see from (A2), the leading-order flow is coupled to the first-order pressure, resulting in the Stokes equations. As we do not require any further results from this region, we proceed straight to Region III.

\section{Region III}

To move from Region II to Region III, we further scale $y=\bar{y} / \bar{\delta}$ and $u=\bar{\delta} \bar{u}$. Using $\epsilon \eta=\bar{\delta} \psi$ again, the governing equations become

$$
p_{\bar{x}}=O\left(\bar{\delta}^{2}\right), \quad p_{\bar{y}}=\frac{\psi^{4} \beta}{\eta^{4}} \bar{v}_{\bar{x} \bar{x}}+O\left(\bar{\delta}^{2}\right), \quad \bar{u}_{\bar{x}}+\bar{v}_{\bar{y}}=0 \quad \text { for }|\bar{x}|<1, \quad y \in(-v \eta / \psi, 0) .
$$

The boundary conditions are

$$
\begin{gathered}
\bar{u}=0, \quad \bar{v}=0 \quad \text { for }|\bar{x}|=1, \quad y \in(-v \eta / \psi, 0), \\
p=0 \quad \text { for }|\bar{x}|<1, \quad y=-v \eta / \psi .
\end{gathered}
$$

The system (A4) is solved by

$$
\bar{u}=0, \quad \bar{v}=\frac{A \eta^{4}}{2 \psi^{4} \beta}\left(\bar{x}^{2}-1\right), \quad p=A\left(\bar{y}+\frac{\nu \eta}{\psi}\right),
$$

where $A$ is an as-of-yet unknown constant. To obtain (45), and the Dirac delta function within, we note that the pressure at $\bar{y}=0$ is given by

$$
p(\bar{x}, 0)=A v \eta / \psi,
$$

and the total dimensionless flux out of the channel (and the system) is given by

$$
-\int_{-1}^{1} \bar{v}(\bar{x},-v / \psi) d \bar{x}=\frac{2 A \eta^{4}}{3 \psi^{4} \beta}
$$

Noting that the flux is conserved within Region III, we can combine (A3), (A6), and (A7) with (43), the solutions for the flow in Region Ib, to deduce the strength of the Dirac delta function in (45), thereby closing the system.

[1] C. C. Michel and F. E. Curry, Microvascular permeability, Physiol. Rev. 79, 703 (1999).

[2] J. M. Tarbell and L. M. Cancel, The glycocalyx and its significance in human medicine, J. Int. Med. 280, 97 (2016).

[3] J. A. Florian, J. R. Kosky, K. Ainslie, Z. Pang, R. O. Dull, and J. M. Tarbell, Heparan sulfate proteoglycan is a mechanosensor on endothelial cells, Circ. Res. 93, 136 (2003).

[4] S. Weinbaum, J. M. Tarbell, and E. R. Damiano, The structure and function of the endothelial glycocalyx layer, Annu. Rev. Biomed. Eng. 9, 121 (2007). 
[5] J. R. Levick and C. C. Michel, Microvascular fluid exchange and the revised Starling principle, Cardiovasc. Res. 87, 198 (2010).

[6] J. R. Levick, An Introduction to Cardiovascular Physiology (Butterworth-Heinemann, London, 2013).

[7] S. Oltean, Y. Qiu, J. K. Ferguson, M. Stevens, C. Neal, A. Russell, A. Kaura, K. P. Arkill, K. Harris, C. Symonds et al., Vascular endothelial growth factor- $\mathrm{A}_{165} \mathrm{~b}$ is protective and restores endothelial glycocalyx in diabetic nephropathy, J. Am. Soc. Nephrol. 26, 1889 (2014).

[8] C. Chelazzi, G. Villa, P. Mancinelli, A. R. De Gaudio, and C. Adembri, Glycocalyx and sepsis-induced alterations in vascular permeability, Crit. Care 19, 26 (2015).

[9] K. Betteridge, K. Arkill, C. Neal, S. Harper, B. Foster, S. Satchell, D. Bates, and A. Salmon, Sialic acids regulate microvessel permeability, revealed by novel in vivo studies of endothelial glycocalyx structure and function, J. Physiol. 595, 5015 (2017).

[10] F. E. Curry and C. C. Michel, The endothelial glycocalyx: Barrier functions versus red cell hemodynamics: A model of steady state ultrafiltration through a bi-layer formed by a porous outer layer and more selective membrane-associated inner layer, Biorheology 56, 113 (2019).

[11] E. M. Landis, Micro-injection studies of capillary permeability: II. The relation between capillary pressure and the rate at which fluid passes through the walls of single capillaries, Am. J. Physiol. 82, 217 (1927).

[12] C. C. Michel, J. C. Mason, F. E. Curry, J. E. Tooke, and P. J. Hunter, A development of the Landis technique for measuring the filtration coefficient of individual capillaries in the frog mesentery, Exp. Physiol. 59, 283 (1974).

[13] R. H. Adamson and G. Clough, Plasma proteins modify the endothelial cell glycocalyx of frog mesenteric microvessels, J. Physiol. 445, 473 (1992).

[14] K. P. Arkill, K. Qvortrup, T. Starborg, J. M. Mantell, C. Knupp, C. C. Michel, S. J. Harper, A. H. J. Salmon, J. M. Squire, D. O. Bates, and C. R. Neal, Resolution of the three dimensional structure of components of the glomerular filtration barrier, BMC Nephrol 15, 24 (2014).

[15] K. P. Arkill, C. Knupp, C. C. Michel, C. R. Neal, K. Qvortrup, J. Rostgaard, and J. M. Squire, Similar endothelial glycocalyx structures in microvessels from a range of mammalian tissues: Evidence for a common filtering mechanism? Biophys. J. 101, 1046 (2011).

[16] K. P. Arkill, C. R. Neal, J. M. Mantell, C. C. Michel, K. Qvortrup, J. Rostgaard, D. O. Bates, C. Knupp, and J. M. Squire, 3D reconstruction of the glycocalyx structure in mammalian capillaries using electron tomography, Microcirculation 19, 343 (2012).

[17] J. Hegermann, H. Lünsdorf, M. Ochs, and H. Haller, Visualization of the glomerular endothelial glycocalyx by electron microscopy using cationic colloidal thorium dioxide, Histochem. Cell. Biol. 145, 41 (2016).

[18] E. E. Ebong, F. P. Macaluso, D. C. Spray, and J. M. Tarbell, Imaging the endothelial glycocalyx in vitro by rapid freezing/freeze substitution transmission electron microscopy, Arterioscler. Thromb. Vasc. Biol. 31, 1908 (2011).

[19] E. H. Starling, On the absorption of fluids from the connective tissue spaces, J. Physiol. 19, 312 (1896).

[20] C. C. Michel, Starling: The formulation of his hypothesis of microvascular fluid exchange and its significance after 100 years, Exp. Physiol. 82, 1 (1997).

[21] X. Hu, R. H. Adamson, B. Liu, F. E. Curry, and S. Weinbaum, Starling forces that oppose filtration after tissue oncotic pressure is increased, Am. J. Physiol.-Heart C 279, 1724 (2000).

[22] R. H. Adamson, J. F. Lenz, X. Zhang, G. N. Adamson, S. Weinbaum, and F. E. Curry, Oncotic pressures opposing filtration across non-fenestrated rat microvessels, J. Physiol. 557, 889 (2004).

[23] S. Weinbaum, Models to solve mysteries in biomechanics at the cellular level; A new view of fiber matrix layers, Ann. Biomed. Eng. 26, 627 (1998).

[24] C. C. Michel, K. P. Arkill, and F.-R. Curry, The revised Starling principle and its relevance to perioperative fluid management, in Perioperative Fluid Management, edited by E. Farag and A. Kurz (Springer, Cham, 2016), pp. 31-74.

[25] X. Hu and S. Weinbaum, A new view of Starling's hypothesis at the microstructural level, Microvasc. Res. 58, 281 (1999). 
[26] X. Zhang, R. H. Adamson, F.-R. Curry, and S. Weinbaum, A 1-D model to explore the effects of tissue loading and tissue concentration gradients in the revised Starling principle, Am. J. Physiol.-Heart C 291, H2950 (2006).

[27] S. Weinbaum, X. Zhang, Y. Han, H. Vink, and S. C. Cowin, Mechanotransduction and flow across the endothelial glycocalyx, Proc. Natl. Acad. Sci. USA 100, 7988 (2003).

[28] L. Facchini, A. Bellin, and E. F. Toro, A time-dependent multi-layered mathematical model of filtration and solute exchange, the revised Starling principle and the Landis experiments, Veins Lymphatics 6, 101 (2017).

[29] M. Sugihara-Seki, T. Akinaga, and T. Itano, Flow across microvessel walls through the endothelial surface glycocalyx and the interendothelial cleft, J. Fluid Mech. 601, 229 (2008).

[30] D. A. Nield and A. Bejan, Convection in Porous Media (Springer, 2006).

[31] D. A. Nield, The Beavers-Joseph boundary condition and related matters: A historical and critical note, Transp. Porous Media 78, 537 (2009).

[32] T. Levy and E. Sanchez-Palencia, On boundary conditions for fluid flow in porous media, Int. J. Eng. Sci. 13, 923 (1975).

[33] G. S. Beavers and D. D. Joseph, Boundary conditions at a naturally permeable wall, J. Fluid Mech. 30, 197 (1967).

[34] P. G. Saffman, On the boundary condition at the surface of a porous medium, Stud. Appl. Math. 50, 93 (1971).

[35] G. Késmárky, P. Kenyeres, M. Rábai, and K. Tóth, Plasma viscosity: A forgotten variable, Clin. Hemorheol. Micro. 39, 243 (2008).

[36] J. Bear, Dynamics of Fluids in Porous Media (Courier Corporation, 2013).

[37] M. P. Dalwadi, S. J. Chapman, J. M. Oliver, and S. L. Waters, The effect of weak inertia in rotating high-aspect-ratio vessel bioreactors, J. Fluid Mech. 835, 674 (2018).

[38] M. D. van Dyke, Perturbation Methods in Fluid Dynamics (Parabolic Press, 1975).

[39] O. Mongin, C. Rouxel, A.-C. Robin, A. Pla-Quintana, T. R. Krishna, G. Recher, F. Tiaho, A.-M. Caminade, J.-P. Majoral, and M. Blanchard-Desce, Brilliant organic nanodots: Novel nano-objects for bionanophotonics, in Nanobiosystems: Processing, Characterization, and Applications, Vol. 7040 (International Society for Optics and Photonics, 2008), p. 704006.

[40] M. Sugihara-Seki, Transport of spheres suspended in the fluid flowing between hexagonally arranged cylinders, J. Fluid Mech. 551, 309 (2006).

[41] J. Fischbarg and F. P. J. Diecke, A mathematical model of electrolyte and fluid transport across corneal endothelium, J. Membr. Biol. 203, 41 (2005).

[42] P. P. Sumets, J. E. Cater, D. S. Long, and R. J. Clarke, Electro-poroelastohydrodynamics of the endothelial glycocalyx layer, J. Fluid Mech. 838, 284 (2018).

[43] M. Dvoriashyna, A. J. E. Foss, E. A. Gaffney, O. E. Jensen, and R. Repetto, Osmotic and electroosmotic fluid transport across the retinal pigment epithelium: A mathematical model, J. Theor. Biol. 456, 233 (2018).

[44] A. Bensoussan, J.-L. Lions, and G. Papanicolaou, Asymptotic Analysis for Periodic Structures (NorthHolland, Amsterdam, 1978). 